Slavica

bruxellensia

\section{Slavica bruxellensia}

Revue polyphonique de littérature, culture et histoire

slaves

$10 \mid 2014$

Espace slave, espace germanique

\title{
Les relations polono-allemandes dans les proses polonaise et allemande après 1945
}

\section{Kornelia Ćwiklak}

Traducteur : Katia Vandenborre

\section{OpenEdition}

\section{Journals}

Édition électronique

URL : http://journals.openedition.org/slavica/1536

DOI : 10.4000/slavica.1536

ISSN : 2034-6395

\section{Éditeur}

Université libre de Bruxelles - ULB

\section{Référence électronique}

Kornelia Ćwiklak, « Les relations polono-allemandes dans les proses polonaise et allemande après 1945 », Slavica bruxellensia [En ligne], 10 | 2014, mis en ligne le 15 avril 2014, consulté le 01 mai 2019. URL : http://journals.openedition.org/slavica/1536 ; DOI : 10.4000/slavica.1536

Ce document a été généré automatiquement le 1 mai 2019.

\section{$(1) \Theta \Theta$}

Les contenus de Slavica bruxellensia sont mis à disposition selon les termes de la Licence Creative Commons Attribution - Pas d'Utilisation Commerciale - Pas de Modification 3.0 France. 


\title{
Les relations polono-allemandes dans les proses polonaise et allemande après 1945
}

\author{
Kornelia Ćwiklak
}

Traduction : Katia Vandenborre

1 Dans les relations polono-allemandes, nous pouvons observer une césure à la fin des années 1980. Les conditions géopolitiques dans lesquelles se trouvent les deux états voisins sont alors modifiées à la suite du démantèlement pacifique du régime socialiste en Pologne résultant des débats de la Table Ronde (du 6 février au 5 avril 1989) et des premières élections parlementaires partiellement libres (le 4 juin 1989) ainsi que de la chute du mur de Berlin (le 10 novembre 1989) et de la réunification de l'Allemagne (le 3 octobre 1990). Elles évolueront de manière diamétralement opposée.

2 Le nombre de travaux précieux et intéressants que les chercheurs polonais et allemands ont à plusieurs reprises publiés en commun démontre que les relations polonoallemandes se sont révélées un sujet de recherche attrayant des deux côtés de l'Oder : Nie bĘdzie nigdy Niemiec Polakowi bratem...? (L'Allemand ne sera jamais un frère pour le Polonais... ?, 1995) sous la direction de Marek Zybura ${ }^{1}$, Polacy i Niemcy. 100 kluczowych pojęć (Les Polonais et les Allemands. 100 concepts clés, 1996) sous la direction d'Ewa Kobylińska, Andreas Lawaty et Stephan Rüdiger², Obcowanie $z$ wolnością. Dialogi literackie polsko-niemieckie (Rapports à la liberté. Dialogues polono-allemands, 1994 et 2001) sous la direction de Bożena ChrzĄstowska et Hans Dieter Zimmermann ${ }^{3}$, Niemcy $w$ Polsce (Les Allemands en Pologne, 2001) par Zybura ${ }^{4}$, Polska-Niemcy. Pogranicze kulturowe $i$ etniczne (Pologne-Allemagne. Les frontières culturelles et ethniques, 2004) sous la direction de Janusz Kamocki, Krystyna Kwaśniewicz et Anna Spis ${ }^{5}$, Polsko-niemieckie miejsca pamiĘci (Les lieux de mémoire polono-allemands, 2012) sous la direction de Robert Traba et Hans Henning Hahn', pour n'en citer que quelques-uns. 


\section{Image des Allemands dans la littérature polonaise d'avant-guerre}

3 Dans la littérature d'avant 1989, l'image des Allemands et de la culture allemande suit deux courants et deux langages: d'une part, celui de la propagande de la Pologne Populaire et, d'autre part, celui de la grande littérature. Les stéréotypes jouent un rôle essentiel dans la perception réciproque des représentants des deux nations: dans le premier cas, ils servent (conformément à la classification de Zofia Mitosek ${ }^{7}$ ) à affermir les représentations hostiles déjà existantes, dans le deuxième ils reposent sur le démasquage et le désarmement des stéréotypes par la littérature.

4 Les années d'après-guerre sont dominées par une image de l'Allemand fortement conditionnée par les expériences négatives de la guerre. L’Allemand est montré comme un «maitre arrogant, turbulent, traitant de haut les autres nationalités, convaincu de sa mission orientale de Kulturträger (porteur de la culture) $»^{8}$. Un tel portrait est visible notamment dans Medaliony (Les Médaillons, 1946) de Zofia Nałkowska. Les images d'Allemands dépeints à l'aide de schémas simplifiés et stéréotypés dominent. Conformément à ces schémas, le métier allemand le plus adéquat semble être «le métier d'assassin » (d'après l'expression de Paul Celan «Der Tod ist ein Meister aus Deutschland »" [La mort est un maître venu d'Allemagne] dans « Todesfuge » [Fugue de la mort, 1952]).

5 Les artistes polonais qui avaient des contacts personnels avec des représentants du monde culturel allemand dans l'entre-deux-guerres ont été particulièrement choqués par les actions des occupants visant à exterminer l'intelligentsia polonaise, en commençant par l'arrestation de près de deux cents professeurs de l'Université Jagellonne de Cracovie au tout début de la guerre (le 6 novembre 1939), qu'il s'agisse de l'exécution de professeurs de l'Université et de l'École Polytechnique de Lvov après le déclenchement de la guerre germano-soviétique (le 4 juillet 1941) ou de la déportation systématique des représentants des couches culturelles dans des camps de concentration, considérées comme des actions conscientes visant à priver les Polonais de leur identité nationale. Dans son livre Życie na niby. PamiĘtnik po klĘsce (La vie en apparence. Mémoires après la défaite, 1939-1940), l'éminent critique Kazimierz Wyka exprime l'immense déception causée par cette "grande nation » dont il ne remettait alors même pas en question la contribution à la culture mondiale. En revanche, il en soulignait l'hypocrisie, celle-là même qui avait empêché les Allemands de comprendre les raisons de leur défaite dans la Première Guerre mondiale : « Dans leurs propres manières d'agir, ils ne sont pas capables de percevoir leur faute et je ne pense pas que ce ne soit dû qu'à leur aveuglement moral. » 10

6 Les nouvelles sur Auschwitz de Tadeusz Borowski proposent une autre perspective. En abordant la problématique du camp de concentration, du bourreau et de la victime dans une perspective universelle, sans se limiter à la critique des Allemands; elles atteignent un niveau artistique élevé, une force d'impact et une crédibilité incroyable. Andrzej Werner a qualifié leur contenu "d'apocalypse ordinaire »"

7 Après la guerre, l'attitude négative par rapport à la culture et à la langue allemande change clairement. Dans les discours de propagande, cela signifie néanmoins que les Allemands commencent à se diviser en «bons » (de l'Allemagne de l'Est) et en « mauvais » (de l'Allemagne de l'Ouest). Les commentateurs et les critiques spécialisés dans la 
littérature allemande (entre autres Jan Koprowski, Egon Naganowski, Marcel Ranicki, Wilhelm Szewczyk, Witold Wirpsza), qui en devinrent également d'excellents traducteurs, commencent à jouer un rôle primordial. Dans ces années 1948-1956, les figures de la littérature allemande classique (Johann Wolfgang von Goethe, Friedrich von Schiller, Heinrich Heine, Gotthold Ephraim Lessing) sont parmi les auteurs les plus souvent traduits et édités.

La littérature dite des Territoires Récupérés est notamment utilisée pour façonner l'image des Allemands à des fins de propagande. Parmi les auteurs les plus importants, il faut citer Eugeniusz Paukszta, un des chroniqueurs et des thuriféraires du « retour des Territoires Récupérés dans la Patrie », qui montre dans ses romans la colonisation des Territoires de l'ouest par les colons de l'est, et ce, sous différents titres évocateurs : Trud ziemi nowej (La peine de la terre nouvelle, 1948), Lody pĘkajĄ (La glace se fend, 1955), Pogranicze (Les confins, 1961), Odzyskane gniazda (Les nids récupérés, 1963), Wrastanie (L'accroissement, 1964), Zawsze z tej ziemi (Toujours de cette terre, 1976). Ce sont des romans sociopolitiques, idéologiquement engagés, qui sont des exemples modèles de la manière dont il est possible de créer une image littéraire de l'Autre basée sur des stéréotypes.

Edward Balcerzan affirme qu'il est mieux d'observer cette « germanité » représentée dans la littérature au travers du prisme de la poétique du texte :

La poétique doit permettre de comprendre la manière dont une œuvre peut se définir par rapport à une culture non indigène (étrangère), et en même temps comment il se fait que la littérature donne au récepteur un sentiment de communion, par exemple avec la langue allemande. ${ }^{12}$

Le personnage littéraire est une figure poétique significative dans la littérature à thématique allemande. Il s'agit en effet d'un Allemand qui est le signe de la culture allemande, le signe de la « germanité ».

La construction des personnages et les schémas narratifs situent les romans de Paukszta dans le courant de la littérature populaire. Nous pouvons leur appliquer avec succès les mots d'Anna Martuszewska: "Le principe fondamental de composition englobant l'ensemble de la structure des œuvres relevant de la littérature populaire est la polarisation du monde qui y est représenté selon un schéma clairement défini comme axiologiquement noir et blanc. $»^{13}$

11 Le roman politique d'espionnage Lody pॄ̨kają est celui qui mobilise le plus les mécanismes de la littérature populaire à des fins de propagande. Il reproduit le schéma narratif du roman d'espionnage déjà développé avant la guerre. L'arrière-fond et le commentaire idéologique ont bien entendu changé ici, mais les rôles des personnages principaux ont été conservés. Parmi les protagonistes, nous avons donc le Héros Invincible (le président de l'organisation du parti de l'usine), la Femme-Ange dans le rôle de la victime exploitée et trompée (l'institutrice de l'école maternelle locale) et le Monstre Démoniaque (inutile de dire que c'est l'espion allemand). Chacun des romans étudiés contient des trames sensationnelles et amoureuses, ce qui trahit la volonté de s'adresser à un lectorat le plus large possible.

12 Le langage, non seulement celui des personnages, mais aussi celui de la narration, suggère la volonté de traduire la conscience d'un " homme simple », d'un ouvrier, d'un paysan qui se trouvait sur les Territoires Récupérés et qui ne comprend pas grand-chose à la réalité qui l'entoure. Il est certainement aussi le destinataire projeté dans le texte. La tentative de décoder la situation communicationnelle se présenterait alors de la manière suivante : 
le destinateur agissant sur ordre des autorités exagère le sentiment de menace de la part des Allemands afin de consolider les anciens et les nouveaux habitants des Territoires Récupérés, faire naitre en eux la conviction qu'ils ne deviennent les propriétaires de ces terres que grâce aux changements de régime en Pologne.

Une telle image des Allemands était répandue dans la littérature populaire des années 1960 et 1970. Néanmoins, des signes de changement dans l'approche de la problématique polono-allemande et des tentatives de réévaluer les jugements historiques font déjà leur apparition à cette époque, en particulier dans la littérature artistique de haut niveau. La poésie de Krzysztof Karasek, surtout le poème « Deutsches Requiem » (Requiem allemand, 1978), en est un exemple ${ }^{14}$. D'autres écrivains remarquables créent des portraits bien plus complexes des Allemands et des relations polono-allemandes dans leurs œuvres, sans succomber aux stéréotypes. Andrzej Kuśniewicz en fait partie avec Eroica (Eroica, la symphonie héroïque,1963), Stan nieważkości (L'état d'apesanteur ${ }^{15}$,1973) et Trzecie królestwo (Le troisième royaume,1975). Le héros et narrateur à la première personne d'Eroica est l'officier SS et aristocrate autrichien Ottokar von Valentin. Son personnage anéantit le stéréotype répandu qui dérive l'hitlérisme de l'esprit prussien.

parait le remarquable roman de Stanisław Grochowiak Trismus. Ici aussi, le narrateur et héros de premier plan est un officier SS, le commandant du camp de Glückauf. Les excès des occupants observés pendant la guerre, c'est ce trismus en titre, c'est-à-dire la brève contraction musculaire qu'ont les chiens enragés quand ils mordent. Le comportement de l'Allemand "infecté » par l'hitlérisme est un réflexe comme le trismus chez un animal enragé. Telle est aussi l'obéissance aveugle du héros vis-à-vis de ses supérieurs. Le livre de Grochowiak tente d'analyser les déterminants psychologiques du nazisme. Sur l'exemple de son héros, il montre les émotions et les motivations d'un partisan d'Hitler. Son apparence émotionnelle et son sentimentalisme montrent clairement qu'il a pleuré non pas quand il a appris la mort de sa femme, mais quand, en entendant les paroles de Himmler, il a été ému par « l'innocence » de la neige.

Le recueil de nouvelles de Henryk Worcell Najtrudniejszy jĘzyk świata (La langue la plus difficile du monde, 1965) fait aussi partie des tentatives intéressantes de surmonter la perception stéréotypée des Allemands. Il propose une vision des contacts polonoallemands sur les Territoires occidentaux juste après la guerre qui diffère de celle de Paukszta. Le rapport du narrateur aux Allemands est ambivalent : il est un mélange de curiosité, de sympathie et d'aversion. Les Allemands travaillent toujours dans leurs exploitations, ils aident les nouveaux propriétaires, les Polonais. Méfiants au début, Kornecki et "son" Allemand, Paul Hattwig, se prennent néanmoins d'affection et s'habituent l'un à l'autre: «Je me sentais tout à fait bien à ses côtés. (...) Mais cet hiver passé ensemble sous un même toit nous a permis de nous apprivoiser. ${ }^{16}{ }^{16}$ 'auteur essaye d'éviter les stéréotypes, tant par rapport aux Polonais que par rapport aux Allemands. L'attitude du narrateur à leur égard est aussi nuancée : il ne ménage pas la malice des Polonais (du lieutenant Stopka, de la bonne femme soldat Hruszczewska). En revanche, Paul et sa sœur Elza sont «doux », Reinhardt est « un gosse adorable »; seule Erna, la femme de Paul, reçoit le surnom de « diablesse». Celle qui éveille le plus de sympathie est la vielle Hattwig: "c'est à ses côtés que j'oubliais le plus facilement que j'avais affaire à une Allemande, il s'en fallait de peu pour que je me sente comme un vacancier aux côtés d'une aubergiste de campagne... $\gg^{17}$ Dans cet ouvrage, les Allemands sont bien entendu perçus dans leur différence, telle qu'enregistrée par le narrateur. Ici, cependant, les Allemands ne font pas peur (comme dans les romans de Paukszta). Le contact réel ne 
confirme pas les pseudo-vérités stéréotypées : les Allemands sont normaux. En fait, le conflit est représenté d'une autre manière. Profondément caché au début, il augmente avec le développement de l'intrigue (la composition du recueil est proche de celle du roman). Malgré les apparences de symbiose irréprochable, les colons polonais ne gratifient pas les Allemands de sympathie en retour :

Enfin, quelque chose de non allemand, un enfant simplement, un pur enfant. Ceuxlà dans la grange, ce sont de vrais Allemands, et ce petit et cette grand-mère... (...) Je sais, j'écrirai que la véritable germanité commence chez ces gens à partir de sept ans et qu'elle se termine vers la soixantaine! Quand ils ne peuvent pas encore et quand ils ne peuvent plus... ${ }^{18}$

L'auteur montre ses héros dans la force de leurs préjugés; quant au narrateur, il le construit de manière à ce que quelque chose « lui ordonne » de céder à l'aversion envers les Allemands et de prononcer des mots dont il aura honte après, mais qui, même s'il les regrette, le fera se sentir dans son droit. Dans Najtrudniejszy jĘzyk świata, une véritable entente avec les Allemands est impossible.

Dans la nouvelle Grunwald de Sławomir Mrożek, la confrontation avec l'image intérieure, profondément enracinée de l'Allemand est entretenue dans une tonalité plus libre. Plusieurs années après la guerre, des ordres («Halt» [halte], «Zurück» [arrière], « Vorwärts » [avant]) prononcés sur un ton énergique, bien que de bonne foi, par un passant allemand pour aider à garer une voiture rappellent unanimement la guerre au narrateur polonais et éveille une certaine aversion.

\begin{abstract}
Halt... zurück... facile à dire pour lui. Halt c'était encore un moindre mal, surtout qu'il n'avait pas ajouté Hände Hoch! (...) Va pour vorwärts. D'autant plus qu'il savait ce qu'il faisait, et moi pas. Impuissant et paniqué, je cédai volontiers à son autorité. Sa connaissance des choses était évidente, mais un souvenir d'il y a... combien ?... quinze, seize ans refit soudain surface, et persistait parallèlement à d'autres actions de mon cerveau, ces «arrière... stop... débrayer... » (...) Les sentiments confus ne sont pas sains, ils témoignent du fait que nous venons d'une situation anormale. C'est encore pire quand il n'y a plus de situation anormale, mais que les sentiments confus restent. ${ }^{19}$
\end{abstract}

17 La littérature de la République Populaire de Pologne, en particulier celle qui s'inspire des facteurs officiels, perpétue les images stéréotypées des Allemands qui existent dans la conscience des lecteurs. Aux anciens stéréotypes du XIX ${ }^{e}$ siècle (Allemand-Chevalier Teutonique), elle en ajoute de nouveaux: Allemand-fasciste et Allemand-révisionniste. Seule la grande littérature s'efforce de s'y opposer, désireuse de créer des conceptions plus approfondies et non schématiques. Le ton antigermanique toutefois dominant (au moins en nombre) dans la littérature des premières décennies d'après-guerre aide à préserver le sentiment de grief historique, à compenser la conscience des pertes territoriales et de la vassalisation de la Pologne, et, par conséquent, il consolide le stéréotype « d'hostilité perpétuelle »

\title{
3. Image des Polonais dans la littérature allemande d'après-guerre
}

Les images de la Pologne et des Polonais dans la littérature allemande d'après-guerre subit des évolutions, comme celle de l'Allemagne et des Allemands dans la littérature polonaise. Elles sont influencées par deux complexes de conscience qui sont liés à l'histoire récente de l'Allemagne et qui suscitent des émotions contradictoires: le 
phénomène de la "culpabilité allemande » (conformément à la terminologie de Karl Jaspers) et l'expérience de la perte de l'Est allemand.

L'historien allemand Klaus Ziemer estime que ce deuxième facteur a été dominant pendant de longues années après la guerre :

Après la défaite totale de l'Allemagne dans la Deuxième Guerre mondiale, l'image de la Pologne (...) n'est pas formée par le décompte des crimes commis par des Allemands en Pologne. Un bien plus grand poids est accordé à la perte des régions de l'Oder et de la Neisse, ce qui pour des millions d'Allemands signifiait la nécessité de quitter leur pays d'origine. Le destin des expulsés était ressenti comme une extrême injustice. ${ }^{21}$

Le nom de propagande des «Territoires Récupérés » était considéré comme une moquerie et ces territoires étaient jugés comme occupés. L'Allemagne de l'ouest n'a pas reconnu la frontière de l'Oder et de la Neisse avant la moitié des années 1960. Par ailleurs, le fait que la Pologne avait perdu presque la moitié de son territoire d'avant-guerre (conformément aux résolutions de Yalta), raflé par l'Union Soviétique, était ignoré. En conséquence, des millions de Polonais avaient été déplacés des anciennes provinces orientales de la République de Pologne (provinces devenues lituaniennes, biélorusses et ukrainiennes dans les républiques soviétiques après la guerre) vers la Pologne dans ses nouvelles frontières. C'était donc une expérience analogue à l'expérience allemande, mais l'opinion publique des deux états allemands y était indifférente. Sa position n'a évolué qu'avec le changement d'attitude des églises allemandes, notamment le mémorandum de l'Église évangélique (1965) postulant le droit à leur pays d'origine pour les Polonais nés sur ces territoires après 1945 (de même que pour les déplacés allemands) et l'échange de lettres entre les évêques polonais et allemands de l'Église catholique. Cela a entraîné un tournant dans la conscience collective, initiant la «nouvelle politique de l'est » du gouvernement de Willy Brandt, comme le fait remarquer Ziemer :

La majorité des citoyens de la République Fédérale a alors pris conscience de la dimension non seulement politique, mais aussi morale des relations polonoallemandes, jusque-là reléguée aux oubliettes (...). Toutefois, seuls quelques milieux et personnes engagés ont commencé à participer à la « digestion du passé », la prise de conscience parfois très douloureuse de ce qui s'est passé en Pologne pendant la Deuxième Guerre mondiale, des crimes que les Allemands y ont commis. ${ }^{22}$

20 L'écrivain et Prix Nobel Günter Grass fait partie de ces personnes qui ont activement soutenu la politique de Brandt. Il accompagnait le chancelier lors de la fameuse scène où celui-ci s'est agenouillé devant le monument de Héros du Ghetto de Varsovie (le 7 décembre 1970), geste interprété comme une confession publique de la culpabilité du peuple allemand et comme un hommage aux Juifs assassinés à Varsovie ainsi qu'à toutes les victimes de la Deuxième Guerre mondiale.

21 Grass est un auteur qui a fait des relations polono-allemandes un des thèmes les plus importants de son œuvre. L'écrivain est considéré comme un critique sévère de la société et de l'histoire allemande, comme un satiriste anarchiste et un iconoclaste qui désarme les idéologies, les légendes nationales, les mensonges de la politiques et de la philosophie par la moquerie, comme un moraliste et, en même temps, comme un chantre de l'existence dans sa forme sensuelle. Cet auteur qui n'épargne pas ses compatriotes parle de la Pologne et des Polonais avec un lyrisme discret (teinté d'une ironie mélancolique) ${ }^{23}$.

Die Blechtrommel (Le Tambour, 1959), son œuvre la plus importante, pénètre et utilise les stéréotypes réciproques des Polonais et des Allemands, que l'auteur montre comme le résultat de préjugés. Il attribue aux Allemands un talent organisationnel et une grande 
importance à leur identité nationale, quoiqu'imprécise en raison de la division séculaire des états allemands, ayant servi de terreau au nationalisme du $\mathrm{xx}^{\mathrm{e}}$ siècle. Il présente les Polonais comme des idéalistes romantiques, attachés au concept de nation, forts dans l'improvisation et peu exigeants dans l'organisation.

L'écrivain affirme que tous ses livres épiques, y compris les plus imposants, sont nés de l'inspiration d'un «moment lyrique ». Ainsi, le point de départ de Die Blechtrommel est le poème «Die polnische Fahne » (Le drapeau polonais). Maria Janion considère ce détail comme très important, car il montre ce que ce "moment lyrique» désintéressé, artistique, polonais représente pour ce chef-d'œuvre de la littérature européenne du $\mathrm{xx}^{\mathrm{e}}$ siècle ${ }^{24}$. L'image de la polonité dans Die Blechtrommel doit être interprétée au travers du prisme de la classification générique de ce roman, qui n'est ni un roman historique, ni un roman politique, mais un roman fantastique. C'est avant tout la création de la figure du héros principal, Oskar, qui le prouve, celui-ci agissant dans l'espace fantastique en lequel Gdańsk se mue sous son pouvoir. Comme le dit Janion, le monde fantasmatique de Grass contient le mythe de la polonité25.

Grass se sert de l'image du patriotisme polonais telle qu'elle s'est formée à l'époque romantique, un patriotisme frôlant la folie. L'écrivain se réfère à cette conception, il l'intensifie en créant une série de biographies fantastiques et souvent grotesques de patriotes-maniaques polonais, comme par exemple le grand-père d'Oskar (Józef Koljaiczek) et son oncle (Wincenty Broński). Grass qualifie les Polonais d'habiles. Il le dit le plus souvent possible, car il admire leur fidélité à leurs idéaux et leur détermination dans la quête de leurs rêves. Don Quichotte, chevalier à l'allure mélancolique, noble maniaque, apparaît à la lumière du crépuscule nocturne au pied de Kutno, menant l'assaut de la cavalerie polonaise contre les chars allemands en septembre 1939 (il faut noter que cette séduisante vision n'a toutefois pas d'équivalent dans la réalité). Il est par ailleurs curieux que Grass accepte l'idéalisme polonais, alors qu'il ne supporte par la philosophie idéaliste allemande. Janion interprète cela comme un effet de son aversion pour l'idéalisme de Georg Wilhelm Friedrich Hegel, qui tend à fondre l'individu dans un tout abstrait, alors que le culte romantique de l'individualité, de l'originalité domine dans l'élan polonais vers son idéal. Les conditionnements esthétiques qui influencent l'écrivain ne sont pas sans importance: l'ironie tragique du destin polonais s'accompagne de mélancolie, ce qui implique que l'admiration de Grass pour la beauté mourante a une motivation esthétique. Comme l'écrit Janion, c'est une beauté mélancolique qui exprime le mythe du Royaume Assassinée 26 .

Dans les œuvres de Grass, Siegfried Lenz ou encore Horst Bienk, on retrouve une image des relations polono-allemandes et une vision des confins communs sans préjugés ni distorsions stéréotypées, et présentés, en plus, dans une prose remarquable, aux problématiques riches et plurivoques et aux valeurs artistiques exceptionnelles. D'importants critiques et chercheurs en littérature, comme par exemple Janion, Koprowski, Bolesław Fac, Adam Krzemiński, Zbigniew Światłowski, sont devenus des amateurs et des popularisateurs de ces écrivains. L'auteur de la trilogie dite de Gdańsk (Le Chat et la souris, Le Tambour, Les Années de chien) a toutefois rencontré un accueil controversé dans la Pologne des années 1980. Certains commentateurs, faisant souvent abstraction des aspects littéraires de ses œuvres, les considéraient comme des discours à caractère politico-idéologique plaidant contre le patriotisme polonais, et entraient en conflit avec l'auteur justement sur ce point. 

étant considérée comme représentative de la littérature allemande de l'Ouest et proche du récepteur polonais grâce à sa position chrétienne. Parmi les écrivains de l'ancienne République démocratique allemande, Christa Wolf aborde les relations que les Allemands entretiennent avec leurs voisins orientaux dans son roman Kindheitsmuster (Trame d'enfance, 1976), dans lequel elle revient dans son Landsberg natal (Gorzów). Johannes Bobrowski en fait de même : ses romans et ses nouvelles rencontrent un accueil favorable en Pologne eu égard à la manière dont il y aborde le sujet des confins germano-slaves, qu'il montre comme une mosaïque pluriethnique extraordinairement riche culturellement (la conception du "divan sarmate ») ${ }^{27}$. La thématique suprême de son œuvre, tant en poésie qu'en prose, est la relation des Allemands avec leurs voisins orientaux.

À l'exception des périodes de crise ou de tournant dans la vie sociopolitique du pays, la Pologne ne se trouvait pas au centre des préoccupations de l'opinion publique en Allemagne de l'ouest, comme l'écrit Ziemer :

Il a fallu attendre l'insurrection de « Solidarność » en 1980 pour que des cercles plus larges de l'opinion publique de République Fédérale d'Allemagne commencent à s'intéresser à la Pologne. Elle a révélé de nouvelles images de la Pologne et a rendu possible l'identification de ce qui était polonais: autrement dit, l'attitude patriotique et anticommuniste des Polonais, leurs liens avec l'Église, leur engagement politique et social, leur désir de liberté, etc. Cela a provoqué une vague de fascination pour la Pologne..$^{28}$

Dans le courant des années 1980, l'image de la Pologne s'est cependant détériorée eu égard à l'importante vague d'émigrés qui a commencé à arriver en Allemagne de l'ouest après l'introduction de l'état de siège en Pologne (le 13 décembre 1981). Certains d'entre eux, en particulier ceux qui avaient reçu une bonne formation et auxquels les gouvernements de l'appareil du pouvoir communiste ne permettaient pas de déployer leurs ailes dans la patrie, sont devenus d'estimables employés, mais d'autres n'ont pas contribué à la bonne réputation de la Pologne.

Depuis le renversement pacifique du régime communiste en Pologne et la réunification de l'Allemagne, activement soutenue par les pouvoirs polonais, les comportements propolonais relèvent, comme l'observe Ziemer, de la political correctness. La question de la frontière pesant sur les relations polono-allemandes « a été définitivement réglée, et un accord de bon voisinage, signé le 17 juin 1991, crée des conditions optimales à l'intensification des contacts. ${ }^{29}$ Prisonnier d'Auschwitz et militant de l'opposition démocratique qui a pendant plusieurs décennies travaillé de manière conséquente au rapprochement entre les deux peuples, Władysław Bartoszewski est un symbole de cette réconciliation qui jouit d'un immense respect en Allemagne. Il l'a exprimé dans un émouvant discours au Bundestag lors du cinquantième anniversaire de la fin de la Deuxième Guerre mondiale, alors qu'il parlait en tant que Ministre polonais des Affaires étrangères.

\section{Relations mutuelles dans les années 1980}

Les relations avec les voisins occidentaux subissent d'évidentes modifications dans les années 1980, tandis que les changements sociopolitiques s'intensifient en Pologne et que la scène littéraire voit apparaitre une nouvelle génération d'écrivains nés dans les années 1950 et 1960 dont le rapport aux expériences historiques est différent de celui de leurs 
parents et grands-parents. Pour ces auteurs, il est devenu clair qu'il faut modifier le regard porté sur les Allemands. Les essayistes prennent pour modèle de relations de voisinage la réconciliation entre l'Allemagne et la France, des pays dont l'histoire commune n'a pas été moins chargée que le passé des Polonais et des Allemands. La réconciliation avec l'Allemagne est perçue comme possible et considérée comme une tâche à accomplir.

Un des indicateurs de ce changement a été, comme le remarque Leszek Szaruga, la parution d'une série d'œuvres abordant l'Holocauste d'une manière nouvelle. Il commence à acquérir une dimension universelle, déjà perçue précédemment, mais masquée par la vivacité du drame des Polonais et par l'ampleur de leur martyrologie de guerre. Les plus importantes de ces œuvres ont paru dans les années 1980 : Umschlagplatz (L'Umschlagplatz, 1988) de Jarosław Marek Rymkiewicz, Zagłada (L'extermination, 1987) de Piotr Szewc, Sublokatorka (Le sous-locataire, 1985), Okna (Les Fenêtres,1985), Zdażyć przed Panem Bogiem (Prendre le bon Dieu de vitesse, 1977) ; entretien avec Marek Edelman, le seul chef de l'Insurrection du Ghetto de Varsovie à avoir survécu) d'Hanna Krall, PoczĄtek (littéralement Le Début, traduit en français sous le titre La jolie Madame Seidenman, 1986) d'Andrzej Szczypiorski, Skrawek czasu (Un morceau de temps, 1987) d'Ida Fink et les essais contenus dans Kamień graniczny (La pierre de frontière, 1994) de Piotr Matywiecki. Ces livres proposent un regard plus large, universalisant, mais cela ne signifie pas qu'ils libèrent les Allemands de leur responsabilité dans l'extermination ou qu'ils essayent de relativiser la faute des Allemands. Ils apportent toutefois de nouveaux éléments au sujet: «ils semblent prudemment accepter son caractère d'expérience irréductiblement universelle, (...) fondamentalement différente de tous les antécédents connus dans l'histoire $\aleph^{30}$. Il est intéressant de signaler ici l'association caractéristique de la problématique polono-allemande avec la problématique juive ${ }^{31}$.

Dans les années 1980, une image de l'Allemand qui se réfère à des attitudes et des actes de personnes concrètes, sans se baser sur des indicateurs politico-idéologiques, s'oppose de plus en plus manifestement à la séparation fermement établie par la propagande entre les bons et les mauvais Allemands ${ }^{32}$. C'est ce qui se produit dans Poczatek de Szczypiorski et dans Hanemann (1995) de Stefan Chwin. Ces livres sont traduits en allemand, ils contribuent ainsi à briser des clichés fermement ancrés et à nouer un dialogue entre Polonais et Allemands. C'est devenu possible grâce à la transposition des expériences historiques de la sphère politique vers la sphère psychologique. Connu en Allemagne sous le titre Die schöne Frau Seidenman (La jolie Madame Seidenman), le roman de Szczypiorski joue un rôle particulier, il devient un bestseller, démontrant pour la première fois avec force que l'entente avec les Polonais est possible au-delà du complexe de l'expérience de la souffrance et de la culpabilité de la guerre.

PoczĄtek fait partie des tentatives largement discutées à cette époque de réconciliation polono-allemande. C'est un livre aussi célèbre que controversé. Dans les colonnes d'Odra, il est critiqué par Andrzej Więckowski qui y demande sans détours comment il se fait que Szczypiorski pardonne aux Allemands pour la Deuxième Guerre mondiale ${ }^{33}$. D'où provient ce point de vue si saisissant? À son avis, le procédé de base appliqué dans Początek est l'utilisation libre d'une série « d'anecdotes autobiographiques se terminant à une époque ultérieure $»^{34}$. Il s'agit d'anecdotes "perverses, ironiques, efficaces et en même temps plates, évidentes $»^{35}$. Ce sont elles qui illustrent « la thèse directrice du livre selon laquelle tout est variable dans la vie, les gens diffèrent et l'histoire est moqueuse, ironique, railleuse $»^{36}$. Ce n'est bien entendu pas une découverte, mais cette affirmation doit servir 
le flou de la responsabilité. Par exemple, le petit Juif caché pendant la guerre par des religieuses devient un Polonais sarmate sujet à l'antisémitisme, le romantique soldat de l'Armée Intérieure est bien sûr quelques années plus tard impliqué dans le mouvement de Solidarność, tandis que le "szmalcownik», le maître chanteur persécuteur de Juifs, fait carrière dans le communisme. D'après Dariusz Nowacki :

La géniale invention de Szczypiorski repose premièrement sur l'étalement du champ du récit (cinq ans c'est trop peu), et deuxièmement sur l'extension des griefs. Les relations polono-juives, judéo-allemandes et le communisme s'insèrent dans les relations polono-allemandes. Il est clair que le discours anticommuniste fonctionne ici comme un baume et un calmant en même temps. ${ }^{37}$

Dans les années 1980, la rhétorique anti-allemande commence aussi à perdre de son sens parce qu'elle est éclipsée par la redécouverte et la verbalisation pour la première fois ouverte du traumatisme lié à la perte des Confins orientaux par la Pologne ainsi que la martyrologie des Polonais à l'est. Des camps voilent des camps. La nouveauté tient au fait de mettre le totalitarisme nazi et le totalitarisme soviétique sur le même pied d'égalité. Il est intéressant de remarquer ici que les Allemands ont mis du temps à accepter une telle conception, soulignant la singularité du fascisme. «L'Allemand Polonais » Peter/Piotr Lachmann (qui est resté en Pologne après la guerre où il est devenu un poète, prosateur et traducteur bilingue) souligne la tendance typiquement allemande à viser la perfection dans chaque domaine. Il estime qu'un des pires traits du caractère national allemand est l'inclination vers l'absolutisme et son Monopolanspruch (exigence de monopole). Même la dispute d'après-guerre entre les historiens allemands (Historikerstreit) n'a pas permis la comparaison des crimes nazis et staliniens, ce qui, à son avis, est la preuve d'un esprit malade de concurrence dont résulte le désir de monopole, même en ce qui concerne « le plus grand crime de tous les temps $»^{38}$. Nous retrouvons des exemples de rapprochement du rôle du nazisme et du communisme dans Krótka historia pewnego żartu (Brève histoire d'une certaine blague, 1991) de Chwin, qui se sert d'un double portrait : Hitler va de pair avec Staline, le totalitarisme brun avec le rouge. Une conception similaire est appliquée dans la nouvelle Stót (La table, 1991) de Paweł Huelle : les parents d'une jeune héroïnenarratrice se disputent pour savoir lesquels étaient les pires, les Allemands ou les Russes :

\footnotetext{
Il s'est pendu - maman rentrait justement dans la pièce avec un plat fumant - car sa conscience avait fini par l'agiter. Si tous les Allemands avaient été agités par leur conscience, ils auraient tous fait la même chose - ajouta-t-elle en mettant les pommes de terre en chemise sur la table - ils auraient tous dû se pendre après ce qu'ils ont fait.

- Et les Soviétiques ?! s'exclama mon père en rejetant ses pelures au bord de l'assiette - et les Soviétiques?

Je savais que c'était le début d'une dispute. Maman avait une peur panique des Allemands et rien ne pouvait la soigner de cette angoisse, alors que mon père attribuait les plus grands maux aux compatriotes de Fëdor Dostojevskij. La frontière invisible passait maintenant par la table (...) et ils étaient tous les deux séparés par celle-ci, comme le pays de leur enfance en trente-neuf, (...) on avait déchiré comme rien le morceau de toile en deux parties, entre lesquelles luisait le fil argenté du Bug. ${ }^{39}$
}

Le rapport aux Allemands et aux Russes fait donc partie de cette première éducation à la maison. Il est aussi un élément de la conception du monde héritée de l'école, conforme au discours de propagande de l'État socialiste :

À l'école, nous avions appris que les Allemands qui voisinaient avec nous étaient bons et étaient nos amis, car ils ne voulaient rien de nous, et que ceux qui ne voisinaient pas avec nous étaient mauvais et n'étaient pas nos amis, car ils 
voulaient nous chasser de nos appartements, de notre école et de la ville en général.

40

Ainsi, au tournant des années 1980 et 1990, un net changement se produit : l'attitude de ressentiment et d'hostilité à l'égard de ce qui est allemand cède la place à un intérêt pour la différence. La nouvelle ouverture dans les relations polono-allemandes après 1989 est accompagnée d'une curiosité réciproque, une découverte (déjà entamée auparavant par un petit nombre d'excellents auteurs, tels que Grass, Lenz et Henryk Bienek) des petites patries communes dans les confins occidentaux (dans la littérature polonaise depuis la fin des années 1980: Huelle, Chwin [Gdańsk], Erwin Kruk, Kazimierz Brakoniecki, Włodzimierz Kowalewski [la Mazurie], Artur D. Liskowacki, Inga Iwasiów [Szczecin], Julian Kornhauser, Henryk Waniek, Stefan Szymutko, Kazimierz Kutz [la Haute Silésie]).

On ne peut non plus ignorer l'importance des « histoires de confins » qui étaient autrefois interdites par la censure et qui, sous la forme de tradition familiale incarnée par les parents et les grands-parents des héros, étaient frauduleusement intégrées à la littérature des petites patries situées sur les territoires occidentaux, patries appelées par certains " patries récupérées ». L'expulsion des terres familiales situées dans les anciens confins orientaux de la Pologne facilitait la compréhension des émotions liées à la perte de la terre natale à l'est par les Allemands et permettait d'observer des parallèles existants entre les destins des Polonais et des Allemands déplacés, et ce, malgré les nombreuses différences.

On trouve des exemples de cette thématique dans des œuvres qui présentent des rencontres entre des habitants présents et des anciens habitants des territoires occidentaux. Opowieść heroiczna (Récit héroïque, 1989) de Mirosław Jasiński et Mirosław Spychalski illustre cette germanité de Wrocław découverte par ses habitants d'aprèsguerre. La nouvelle Srebrny deszcz (Pluie d'argent, 1996) de Huelle ainsi que Krótka historia pewnego żartu et Hanemann de Chwin présentent le passé de Gdańsk, stratifié à la manière d'un palimpseste. Dans ces livres, les trames « des confins » s'entrechoquent avec les trames « post-allemandes ». Les livres Miasto-ja-miasto (Ville-moi-ville, 1998) de Iwasiów, Ulice Szczecina (Les rues de Szczecin, 1995), Cukiernica pani Kirsch (Le sucrier de Madame Kirsch, 1998), Eine kleine... (Une petite..., 2000) de Liskowacki révèlent le passé compliqué de Szczecin. Dans ces livres, le complexe de la perte qu'éprouve la génération des déplacés, qui sont montrés comme des mèches déracinées, est remplacé par une progressive appropriation de ces territoires en possession de la génération des enfants qui sont nés là et qui créent une littérature de ré-enracinement. Ce ne sont donc que les écrivains nés dans les années 1950 et 1960 qui ont commencé à se mesurer à l'héritage laissé par les Allemands, et non les déplacés d'après-guerre dont les problèmes dominants étaient le bouleversement politique et personnel, l'immensité des dégâts et la nécessité de reconstruire le pays à partir de zéro.

La littérature des années 1990, dite du courant des petites patries, tente de sauver le souvenir des habitants des confins, de cette région "entre " deux pays ainsi que leur culture spécifique, polono-allemande de transition. Cette littérature ouvre une perspective qui s'éloigne des conditionnements historiques, permettant d'interpréter autrement le concept de nationalité, comme le fait par exemple Andrzej Zawada dans son essai Brestaw (1996), dont le titre est formé par l'assemblage des noms allemand et polonais de la ville ${ }^{41}$. La catégorie du lieu est devenue un facteur primordial pour définir sa propre identité, surtout l'identité basée sur l'identification régionale, et non nationale ou étatique. Ceci est particulièrement important par rapport à l'expérience de la 
Deuxième Guerre mondiale qui a imposé de voir l'histoire comme une grande destructrice des communautés locales. De manière assez caractéristique, dans la littérature des petites patries, la géographie vainc l'histoire. Comme le fait remarquer Szaruga, cette « sortie de l'histoire » dans le domaine de «la terre qui n'appartient à personne » est devenue une bonne base pour surmonter ce qui a été inculqué par la propagande et pour former au sentiment d'aliénation ${ }^{42}$. Cette anhistoricité particulière peut s'observer dans les romans Weiser Dawidek (Weiser David, 1987) de Huelle, Hanemann de Chwin ainsi que dans certains romans d'Olga Tokarczuk. Dans ceux-ci, l'histoire devient un costume conventionnel cachant des problèmes plus importants qui se jouent autour de l'identité propre. Au centre de l'attention, se trouvent les villes et les régions des confins, comme Gdańsk, la Silésie, la Mazurie, qui sont présentées comme les pays des premières initiations.

Grâce à la variété des façons dont elles explorent la thématique allemande, les œuvres plus tardives de la littérature polonaise démontrent qu'il n'y a déjà plus de limite. Dans le roman Ślady na piasku (Des Traces sur le sable, 1994) de Marek JastrzĘbiec-Mosakowski, par exemple, la politique, les questions nationales et l'histoire ne comptent pas, car les héros vivent d'amour et d'art. Kowalewski adopte une perspective similaire dans sa nouvelle Powrót do Breitenheide (Retour à Breitenheide, 1997). L'ancienne Prusse orientale n'est plus montrée que comme un attrayant décor où se joue un drame érotique universel (Ślady na piasku), elle devient un décor «exotique», propice à la réflexion sur l'inéluctabilité du temps qui passe (Powrót do Breitenheide). Il en est pratiquement de même dans la prose d'Ewa Schilling (Akacja [L'Acacia, 2001]), dont l'action se déroule à la limite de la Warmie et de la Mazurie dans la petite ville d'Alsten (Orłowo) dans les années 1945-1956. Elle devient un voyage littéraire dans le passé, mais aussi dans « l'altérité ». Le problème de la quête d'identité des Allemands qui sont restés (lesdits autochtones) et qui sont donc confrontés à une nouvelle réalité, leur relation avec leurs nouveaux voisins, des personnes déplacées venant de régions situées au-delà du Bug, est toutefois mis en relation avec la différence d'orientation sexuelle. Ici, la germanité n'est donc qu'un des nombreux signes de différence. Cette approche extraordinairement large de la thématique allemande dans la littérature polonaise des années 1990 prouve que la réconciliation a déjà eu lieu, et que la thématique allemande a été transposée dans la sphère privée. Ces remarques concernent cependant la prose artistique, et non populaire, ce dont témoigne le roman Niemiecki taniec (La Danse allemande, 2000) de Maria Nurowska. Elle y parle du drame de l'expulsion et se base sur la simple idée du renversement des rôles du bourreau et de la victime.

Des effets très intéressants ont pu également être obtenus par l'application dans plusieurs œuvres du regard sur le monde de «l'autre » depuis l'intérieur, c'est-à-dire la création par l'écrivain polonais d'un narrateur allemand, grâce auquel le lecteur polonais peut avoir un aperçu d'une mentalité et d'une culture qui diffère de la sienne, et en même temps percevoir sa propre culture avec des yeux étrangers, d'une certaine façon de l'extérieur. Ce type de narration est présent entre autres dans Eroica de Kuśniewicz, Hanemann de Chwin, E.E. de Tokarczuk, Powrót do Breitenheide de Kowalewski. Comme le fait remarquer Balcerzan :

Dans les portraits littéraires de la culture allemande, dans ses appréciations polonaises, les opérations artistiques liées à la figure du destinateur se distinguent tout particulièrement (...). Qui est le narrateur : un Polonais ou un Allemand ? C'est de cela que dépend la langue de l'œuvre, la langue des expériences et des valeurs personnelles ou étrangères. ${ }^{43}$ 
Balcerzan appelle de telles œuvres des apocryphes allemands, qu'il définit comme des «tentatives de regarder les Allemands avec le regard d'Allemands inventés par des auteurs polonais $»^{44}$. Il est néanmoins significatif que les identités des héros de ces livres ne s'épuisent ni dans la " germanité », ni dans la " polonité ».

Comme l'observe Nowacki, les écrivains et les intellectuels du cercle "Borussia » d'Olsztyn nous convainquent de l'existence (au moins dans une dimension mythologique) d'une Atlantide du Nord. De la même manière, les voyages de l'essayiste de Waniek dans une contrée du nom de ŚlĄsk-Silésie-Schlesien, en ignorant les circonstances historiques, créent un pays mythique, invalidant ainsi les litiges autour de son appartenance territoriale. Dans ses esquisses, apparaît la conviction qu'il existe « un droit souverain de la terre, ses propres lois, ses attentes et ses destinations $»^{45}$.

41 Liskowacki, l'auteur des œuvres autour de Szczecin précédemment évoqués, a publié en 2002 un recueil sous le titre éloquent Pożegnanie miasta (L'Adieu à la ville), faisant comprendre qu'il ne voulait plus être l'otage d'un seul sujet. Dans un esprit de réconciliation, il avait suffisamment abordé le passé familial allemand de la ville. Il a en même temps signalé que le sujet avait été épuisé.

\section{Pour conclure}

À partir des années 1980, nous pouvons observer une nouvelle vague d'émigration polonaise en Allemagne: ce sont des écrivains qui s'y sont installés soit en tant qu'émigrés politiques, comme par exemple Ewa Maria Slaska, soit en tant que «déplacés tardifs », comme par exemple Stanisław Bieniasz, Janusz Rudnicki, Krzysztof Maria Załuski. Dans leurs œuvres, persiste une intense confrontation de la polonité et de la germanité, ce qui est visible dans les nouvelles de Rudnicki dans son recueil Można żyć (On peut vivre, 1992) ainsi que dans la nouvelle Też (1996) de Załuski, dont la narratrice est la fille d'un déplacé s'efforçant de se retrouver entre deux milieux culturels et linguistiques. Rudnicki, quant à lui, déconstruit les motifs tabous dans les décennies d'après-guerre (par exemple le tatouage des numéros de camp, les images d'extermination dans la langue du flirt). Il démontre qu'ils sont des signes morts depuis longtemps dans l'iconographie martyrologique.

43 Il vaut également la peine d'évoquer les jeunes auteurs qui écrivent en allemand, tels que Dariusz Muszer qui aborde les problèmes polono-judéo-sorabo-allemands dans son livre Die Freiheit riecht nach Vanille (La liberté sent la vanille, 1999) ou encore Artur Becket qui se réfère aux relations polono-mazuro-allemandes dans Der Dadajsee (Le lac de Dadaj, 1997). L'œuvre de Radek Knapp, qui vit en Autriche, mérite une attention particulière : ses livres Franio (1994) et Herrn Kukas Empfehlungen (Les recommandations de Monsieur Kuka, 1999) ont été rapidement traduits en polonais, et le deuxième a fait l'objet d'une adaptation cinématographique réussie. Dans le cas des émigrés, soit ils s'attachent à leur identité polonaise, cultivant la langue, les traditions et le pays d'origine, soit ils choisissent l'identité allemande pour s'adapter à leur nouvel environnement, mais c'est un processus complexe dont plusieurs des écrivains cités illustrent les méandres.

Il est difficile de prédire dans quelle direction la représentation des nations voisines, de leurs représentants et de leurs cultures évolueront dans les deux littératures. Toutefois, il faut noter que les relations officielles entre la Pologne et l'Allemagne n'ont jamais été aussi bonnes, que les deux états collaborent étroitement dans le cadre de l'Union 
Européenne, faisant partie de ses représentants les plus actifs, et que la coopération interrégionale se développe. Cela ne signifie cependant pas que tout a été fait en termes de compréhension et de rapprochement, même si certains auteurs ressentent une certaine surabondance de la thématique allemande, allant jusqu'à parler de « kitsch de la réconciliation ». Il est plus difficile d'éliminer les préjugés profondément ancrés chez les gens ordinaires que de changer l'attitude réciproque des élites artistiques et politiques.

\section{BIBLIOGRAPHIE}

Balcerzan E., « Przygoda trzecia: apokryfy niemieckie » (Troisième aventure : les apocryphes allemands), in : Idem, Przygody człowieka książkowego (ogólne i szczególne) (Les aventures de l'homme du livre [en général et en détail]), PEN, Varsovie, 1990, pp. 30-50.

Celan P., «Todesfuge » und andere Gedichte (« Fugue de la mort » et autres poèmes), Suhrkamp Verlag GmbH, Berlin, 2004.Grözinger E., « “Trójkąt” polsko-niemiecko-żydowski jako temat polskiej literatury współczesnej » (Le Triangle polono-germano-juif comme sujet de la littérature polonaise contemporaine), in : Obcowanie z wolnością. Dialogi literackie polsko-niemieckie (Rapports à la liberté. Dialogues polono-allemands), t. 1 (sous la direction de ChrzĄstowska B.

\& Zimmermann H. D.), Nakom, Poznań/Berlin, 1994, pp. 177-190.

Huelle P., Opowiadania na czas przeprowadzki (Récits pour le déménagement), PULS, Londres, 1991.Janion M., Günter Grass i polski Pan Kichot (Günter Grass et le Don Quichotte polonais), Słowo/ Obraz Terytoria, Gdańsk, 1999.

Jasiński M. \& Spychalski M., Opowieść heroiczna (Récit héroïque), Kret, Wrocław, 1989.

LachmannP., Wywołane z pamięci (Éveillés de la mémoire), Borussia, Olsztyn, 1999.

Martuszewska A., « Topika literatury obiegów popularnych » (La topique de la littérature des mouvements populaires), in : Stownik literatury polskiej XX wieku (Dictionnaire de la littérature polonaise du XXe siècle), sous la direction de Brodzka A. et alii, Ossolineum, Wrocław,1993, p. 1105.

Mitosek Z., Literatura i stereotypy (La littérature et les stéréotypes), Ossolineum-PAN, Wrocław, 1974

Mrożek Sł., Dziennik powrotu (Journal du retour), Noir sur Blanc, Varsovie, 2000.

Nie bĘdzie nigdy Niemiec Polakowi bratem...?(L'Allemand ne sera jamais un frère pour le Polonais... ? ), sous la direction de Zybura M., Okis, Wrocław, 1995, 299 p.

Nowacki D., «O polsko-niemieckim pojednaniu (w literaturze) » (La réconciliation polonoallemande [dans la littérature]), in : Normalność $i$ konflikty. Rozważania o literaturze i życiu literackim w nowych czasach (La normalité et les conflits. Considérations sur la littérature et la vie littéraire des temps nouveaux), sous la direction de Czapliński Prz. \& Śliwiński P., Poznańskie Studia Polonistyczne, Poznań, 2006, pp. 219-233.

Obcowanie z wolnością. Dialogi literackie polsko-niemieckie (Rapports avec la liberté. Dialogues polono-allemands), t. 2 (sous la direction de ChrzĄstowska B.), Nakom, Poznań/Berlin, 2001, $331 \mathrm{p}$.

Polacy i Niemcy. 100 kluczowych pojęć (Les Polonais et les Allemands. 100 concepts clés), sous la direction de Kobylińska E., Lawaty A. \& Rüdiger St., Więzi, Varsovie, 1996.

Polska-Niemcy. Pogranicze kulturowe i etniczne (Pologne-Allemagne. Les frontières culturelles et ethniques), sous la direction de Kamocki J., Kwaśniewicz K. \& Spis A., PTL, Wrocław, 2004. 
Polsko-niemieckie miejsca pamiĘci (Les lieux de mémoire polono-allemands), sous la direction de Traba R. \& Hahn H. H., t. 3, Scholar, Varsovie, 2012.

Szaruga L., « Od stereotypów do wyzwolenia z historii » (Des stéréotypes à la libération de l'histoire), in : Polonistyka, nº 3/1999.

Waniek H., Opis podróży mistycznej z OświĘcimia do Zgorzelca 1257-1957 (Description d'un voyage mystique d'Oświęcim à Zgorzelec 1257-1957), Znak, Cracovie, 1996.

Werner A., Zwyczajna apokalipsa: Tadeusz Borowski i jego wizja świata obozów (L'apocalypse ordinaire : Tadeusz Borowski et sa vision du monde des camps), Czytelnik, Varsovie, 1981. WiĘckowski A., « Szczypiorski w Niemczech » (Szczypiorski en Allemagne), in : Odra, nº 9/2000. Worcell H., Najtrudniejszy jĘzyk świata (La langue la plus difficile du monde), Wydawnictwo ŚlĄsk, Katowice, 1965.

Wyka K., Życie na niby. PamiĘtnik po klĘsce (La vie en apparence. Mémoire après la défaite), Wydawnictwo Literackie, Cracovie-Wrocław, 1984.

Ziemer Kl., « Das deutsche Polenbild der letzten 200 Jahre » (L'image allemande des Polonais ces deux cents dernières années), in : Mythen und Stereotypen auf der beiden Seiten der Oder (Mythes et stéréotypes des deux côtés de l'Oder), sous la direction de Hans Dieter Zimmermann, Forum Guardini, Berlin, 2000.

Zybura M., Niemcy w Polsce (Les Allemands en Pologne), Wydawnictwo DolnoślĄskie, Wrocław, 2001

1.

\section{Introduction}

\section{NOTES}

1. Nie b̨̨dzie nigdy Niemiec Polakowi bratem...?(L'Allemand ne sera jamais un frère pour le Polonais... ?), sous la direction de Zybura M., Okis, Wrocław, 1995, 299 p.

2. Polacy i Niemcy. 100 kluczowych pojęć (Les Polonais et les Allemands. 100 concepts clés), sous la direction de Kobylińska E., Lawaty A. \& Rüdiger St., WiĘzi, Varsovie, 1996, 491 p.

3. Obcowanie z wolnościĄ. Dialogi literackie polsko-niemieckie (Rapports à la liberté. Dialogues polonoallemands), t. 1 (sous la direction de ChrzĄstowska B. \& Zimmermann H. D.), Nakom, Poznań/ Berlin, 1994, 213 p., et t. 2 (sous la direction de ChrzĄstowska B.), Nakom, Poznań/Berlin, 2001, $331 \mathrm{p}$.

4. Zybura M., Niemcy $w$ Polsce (Les Allemands en Pologne), Wydawnictwo Dolnośląskie, Wrocław, 2001, 256 p.

5. Polska-Niemcy. Pogranicze kulturowe i etniczne (Pologne-Allemagne. Les frontières culturelles et ethniques), sous la direction de Kamocki J., Kwaśniewicz K. \& Spis A., PTL, Wrocław, 2004, 333 p.

6. Polsko-niemieckie miejsca pamiĘci (Les lieux de mémoire polono-allemands), sous la direction de Traba R. \& Hahn H. H., t. 3, Scholar, Varsovie, 2012, 472 p.

7. Mitosek Z., Literatura i stereotypy (La littérature et les stéréotypes), Ossolineum-PAN, Wrocław, 1974, 193 p. L'auteur distingue trois types de relation entre la littérature et les stéréotypes : la stabilisation littéraire du stéréotype, la création de stéréotypes littéraires et le décryptage du stéréotype. L'engagement déterminé des stéréotypes leur donne le rôle de signes de connivence entre le destinateur et le destinataire de l'œuvre ou d'outil d'influence du texte (de relais des contenus idéologiques désirés), voire d'objet de démasquage artistique (ils deviennent alors l'objet d'une opération créatrice ayant pour objet la dégradation des idées reçues sur la réalité).

8. Szaruga L., "Od stereotypów do wyzwolenia z historii » (Des stéréotypes à la libération de l'histoire), in : Polonistyka, nº 3/1999, p. 144. 
9. Celan P.,«Todesfuge» und andere Gedichte («Fugue de la mort » et autres poèmes), Suhrkamp Verlag GmbH, Berlin, 2004, p. 12. Le poème «Fugue de la mort» de Paul Celan a paru pour la première fois dans le recueil Der Sand aus den Urnen(Le sable des urnes, 1948); sa deuxième édition, qui a eu un grand retentissement, a paru dans le recueil poétique Mohn und Gedächtnis (Le pavot et la mémoire, 1952).

10. Wyka K., Życie na niby. PamiĘtnik po klĘsce (La vie en apparence. Mémoire après la défaite), Wydawnictwo Literackie, Cracovie-Wrocław, 1984, p. 235.

11. Werner A., Zwyczajna apokalipsa: Tadeusz Borowski i jego wizja świata obozów (L'apocalypse ordinaire : Tadeusz Borowski et sa vision du monde des camps), Czytelnik, Varsovie, 1981, 256 p.

12. Balcerzan E., «Przygoda trzecia: apokryfy niemieckie » (Troisième aventure : les apocryphes allemands), in : Idem, Przygody człowieka książkowego (ogólne i szczególne) (Les aventures de l'homme du livre [en général et en détail]), PEN, Varsovie, 1990, pp. 30-50.

13. Martuszewska A., « Topika literatury obiegów popularnych » (La topique de la littérature des mouvements populaires), in : Stownik literatury polskiej XX wieku (Dictionnaire de la littérature polonaise du XXe siècle), sous la direction de Brodzka A. et alii, Ossolineum, Wrocław,1993, p. 1105.

14. Karasek Krz., Prywatna historia ludzkości (L'histoire privée de l'humanité), Wydawnictwo Literackie, Cracovie, 1978, pp. 48-55.

15. Traduit sous ce titre en 1979 par Christophe Jeżewski et Dominique Autrand (éditions Albin Michel. Paris). [NDlE]

16. Worcell H., Najtrudniejszy jĘzyk świata (La langue la plus difficile du monde), Wydawnictwo ŚlĄsk, Katowice, 1965, p. 264.

17. Ibid., p. 294.

18. Ibid., p. 302.

19. Mrożek Sł., Dziennik powrotu (Journal du retour), Noir sur Blanc, Varsovie, 2000, pp. 134-136.

20. J'ai écrit plus amplement sur le sujet dans : «Bliscy nieznajomi. Proza polsko-niemieckiego pogranicza wobec narodowych stereotypów » (Proches inconnus. La prose polono-allemande des confins par rapport aux stéréotypes nationaux), in : Obcowanie z wolnościĄ, op. cit.,t. 2, pp. 191-206.

21. Ziemer Kl., « Niemiecki obraz Polaków w ostatnich dwustu latach » (L'image allemande des Polonais ces deux cents dernières années), in : Obcowanie z wolnościĄ, t. 2, op. cit., p. 131.

22. Ibid., p. 133.

23. Światłowski Zb., "Günter Grass ", in: Pisarze niemieckojĘzyczni XX wieku (Les écrivains de langue allemande du xx ${ }^{\mathrm{e}}$ siècle), PWN, Varsovie-Wrocław, 1996, p. 106.

24. Janion M., Günter Grass i polski Pan Kichot (Günter Grass et le Don Quichotte polonais), Słowo/ Obraz Terytoria, Gdańsk, 1999, p. 58. D’après le critique Mariusz Cieślik, ce livre «montre que Maria Janion a mené à bien son projet de faire de l'écrivain "le maillon manquant de la littérature polonaise". [...] Parmi les écrivains allemands, aucun ne comprend ni ne connaît les Polonais comme Günter Grass, mais aucun n'est aussi compris et adoré chez nous. » : CieślikM., «Günter Grass i polski Pan Kichot, Janion, Maria » [Günter Grass et le Don Quichotte polonais, Janion, Maria], in: Gazeta Wyborcza, 19 janvier 2001. URL: http:// wyborcza.pl/1,75517,108019.html (consulté le 16 octobre 2013).

25. Janion M., Ibid., p. 67.

26. Ibid., pp. 72-73.

27. Chez Johannes Bobrowski, le «tapis sarmate » est une référence manifeste au West-östlicher Divan (Le Divan occidental-oriental,1819) de Johann Wolfgang Goethe qui a déterminé la perception de l'Orient dans la culture allemande pendant de nombreuses années. Toutefois, «l'Orient » désigne ici les pays du Proche-Orient, avant tout la Perse des contes de fées avec sa riche tradition littéraire. On retrouve des échos de l'Orient tel que conçu par Goethe dans les cycles poétiques de Bobrowski Sarmackie czasy (L'époque sarmate, 1961) et Schattenland Ströme 
(Les courants du pays de l'ombre, 1962), aussi appelés « divan sarmate ». Les références à l'œuvre de son grand prédécesseur ont cependant un caractère polémique chez Bobrowski. L'Orient de ce ;io-ci est situé ailleurs : il s'agit du territoire frontalier de la Prusse orientale, de la Pologne et de la Lituanie, fascinant par la multiculturalité. La portée idéologique des deux cycles poétiques est, elle aussi, différente. La conception littéraire de la Sarmatie comprend les paysages perdus de l'enfance, souligne l'importance de la nature et l'inspiration du décor des régions situées audelà du Niémen, évoque les lointains ancêtres polonais, suggère d'approfondir la pluridimensionnalité de sa propre origine, de l'identité tissée de fils allemands et polonais, elle se rapporte également à la question de la culpabilité et de la possibilité d'une compensation poétique. La Sarmatie de Bobrowski est une tentative d'invoquer le mythe de l'Europe de l'est, compris comme un lieu exceptionnel sur la carte du continent, qu'il enrichit par son ensemble particulier de relations historiques, géographiques, naturelles et culturelles.

28. Ziemer Kl., art. cit., p. 134.

29. Idem.

30. Szaruga L., art. cit., pp. 145-146.

31. Voir : Grözinger E., " "TrójkĄt” polsko-niemiecko-żydowski jako temat polskiej literatury współczesnej» (Le Triangle polono-germano-juif comme sujet de la littérature polonaise contemporaine), in : Obcowanie z wolnościĄ, op. cit., t. 1, pp. 177-190.

32. Szaruga L., art. cit., p. 146.

33. WiĘckowski A., "Szczypiorski w Niemczech» (Szczypiorski en Allemagne), in : Odra, $\mathrm{n}^{\circ}$ 9/2000, p. 14.

34. Nowacki D., «O polsko-niemieckim pojednaniu (w literaturze)» (La réconciliation polonoallemande [dans la littérature]), in : Normalność $i$ konflikty. Rozważania o literaturze $i$ życiu literackim $w$ nowych czasach (La normalité et les conflits. Considérations sur la littérature et la vie littéraire des temps nouveaux),sous la direction de Czapliński Prz. \& Śliwiński P., Poznańskie Studia Polonistyczne, Poznań, 2006, pp. 219-233.

35. Idem.

36. Idem.

37. Idem.

38. LachmannP., Wywołane z pamiĘci (Éveillés de la mémoire), Borussia, Olsztyn, 1999, p. 164. « Mistrzostwo świata Niemiec » (le championnat du monde de l'Allemagne), cette citation traduite par Peter/Piotr Lachmann du poème de Celan «La Fugue de la mort » traduit bien le caractère des aspirations allemandes.

39. Huelle P., Opowiadania na czas przeprowadzki (Récits pour le déménagement), PULS, Londres, 1991, p. 8.

40. Jasiński M. \& Spychalski M., Opowieść heroiczna (Récit héroöque), Kret, Wrocław, 1989, p. 4.

41. Ce néologisme est effectivement formé par l'association des deux noms utilisés pour désigner une seule et même ville : Breslau en allemand et Wrocław en polonais. (NdT)

42. Szaruga L., art. cit., p. 150.

43. Balcerzan E., op. cit., p. 24.

44. Idem.

45. Waniek H., Opis podróży mistycznej z OświĘcimia do Zgorzelca 1257-1957 (Description d'un voyage mystique d'Oświęcim à Zgorzelec 1257-1957), Znak, Cracovie, 1996, p. 32. 


\section{RÉSUMÉS}

Le présent article est consacré aux relations polono-allemandes dans les proses polonaise et allemande après 1945. La première partie se focalise sur la représentation des Allemands dans la littérature polonaise entre 1945 et 1989. Deux courants sont alors en présence : d'une part, la propagande officielle de la Pologne Populaire (Eugeniusz Paukszta notamment) et, d'autre part, la grande littérature (Andrzej Kuśniewicz, Stanisław Grochowiak). Dans le premier cas, les stéréotypes jouent un grand rôle dans la perception réciproque, alors que le second tend à les dépasser. La deuxième partie présente des exemples choisis d'images de la Pologne et de Polonais issus la littérature allemande d'après-guerre. L'une des tendances les plus intéressantes a la particularité de s'écarter des préjugés dominants (le Groupe 47: Günter Grass, Johannes Bobrowski et Siegfried Lenz). La section suivante est consacrée à la nouvelle étape qui émerge après 1989, les relations polono-allemandes se caractérisant alors par une curiosité réciproque, une découverte (déjà entamée par quelques écrivains allemands, tels que Günter Grass et Henryk Bienek) des petites patries communes dans les confins occidentaux de la Pologne. Dans la littérature polonaise, cette thématique est explorée à partir de la fin des années 1980 entres autres par Paweł Huelle, Stefan Chwin (Gdańsk), Erwin Kruk, Kazimierz Brakoniecki, Włodzimierz Kowalewski (la Mazurie), Artur D. Liskowacki, Inga Iwasiów (Szczecin), Julian Kornhauser, Henryk Waniek, Stefan Szymutko, Kazimierz Kutz (la Haute-Silésie). Enfin, nous soulevons un dernier aspect: l'œuvre des émigrés polonais vivant en Allemagne. Les représentants de l'ancienne génération écrivent en polonais (Janusz Rudnicki, Stanisław Bieniasz), tandis que les plus jeunes (Radek Knapp, Dariusz Muszer) écrivent en allemand, exprimant dans cette langue une identité polonaise spécifique confrontée à l'environnement allemand ou autrichien dans lequel ils vivent.

\section{INDEX}

Mots-clés : écrivains polonais en Allemangne, littérature allemande, littérature polonaise, prose polonaise, relations polono-allemandes, stéréotypes et leur dépassement

Index géographique : Allemagne, Danzig, Gdańsk, Mazurie, Pologne, Silésie, Stettin, Szczecin Index chronologique : communisme, époque contemporaine, Guerre froide, post-communisme, XXe siècle

Schlüsselwörter : deutsche Gegenwartsprosa, nationale Stereotypen und ihre Überwindung, polnisch-deutsche Beziehungen in den beiden Literaturen dargestellt, polnische Gegenwartsprosa

\section{AUTEURS}

\section{KORNELIA ĆWIKLAK}

Université Adam Mickiewicz de Poznań (Pologne). cwiklak@amu.edu.pl 